\title{
Reducing door to needle time for stroke thrombolysis
}

\author{
Sumanjit Gill \\ West Herts NHS Trust, England
}

\begin{abstract}
Better outcomes are obtained with stroke thrombolysis the more rapidly it is given, both in terms of the patient's level of functional ability and also mortality. Current UK performance targets (outside London) aim for a time of 45 minutes or less. Thrombolysis pathways involve multidisciplinary working across departmental boundaries as well as senior level decision making. Our system used telemedicine out of hours adding additional complexity to the pathway. The initial planning stages started by auditing current practice and mapping the existing pathway. The figures for door to needle times were held on a database on the stroke unit and collected in detail for the purposes of national reporting.

The pathway was mapped by combining personal experience of working within the stroke service with the experiences of the general medical registrars who worked the system out of hours. The initial action was to present this information throughout the hospital at departmental meetings. Opinions were canvassed at these meetings on where the biggest barriers were within the pathway and how we could address them. An awareness campaign was held by advertising over the intranet. An intervention comprising the following elements was introduced over the period of a year: introduction of an ambulance pre-alert, revision of the existing pathway, and education to all those involved in thrombolysis. The cases where particularly long delays were noted were audited in more depth to identify barriers to flow through the system. This was reported in ward meetings for staff to contribute experience and to offer solutions. We went to commissioning group meetings to gain the support of the local ambulance service, and talked to A\&E seniors about the project and the ways in which they could help. Median times were calculated from a stroke database.
\end{abstract}

There was a fall in median door to needle time of 65.5 to 49 minutes over a period of 18 months. A complex intervention to improve door to needle time can and did produce good results with a reduction in times to within the London performance standards of care. The efficacy could be improved with an increased staffing level and provision of a formal setting within which to discuss changes and to keep up the momentum of change.

\section{Problem}

This project was carried out in a district general hospital in a semi rural area of England. The trust serves a catchment area of 100,000 and the stroke service thrombolyses approximately 100 strokes per year (this number rises every year).

The "door to needle times" for thrombolysis are published for each region and our service was not performing as well as other trusts in the area. It was also evident from working within the service that there was some confusion and dissatisfaction about the pathway. In addition to the stroke team, it is staffed by the general medical registrars as well as accident and emergency consultants with stroke specific skills. This causes a problem when trying to provide consistency across the service as well as providing clarity as to who should be called when an acute stroke patient arrives since the rota is so complex. Stroke physician cover is provided by telemedicine out of hours. The figures were audited and a service improvement project was undertaken to reduce the door to needle time within our stroke service.

\section{Background}

Thrombolysis has been shown to improve stroke outcomes both by reducing mortality and disability,[1], and it is most effective the earlier it is administered. One of the ways of measuring the performance of an acute stroke service is by measuring the time within which it is administered. As the service may be staffed by those who are junior or generalists with provision of senior cover by telephone, on site, or by telemedicine, the logistics of providing a continuously effective service can be a challenge to both institute and maintain. However, it is possible to consistently reduce the door to needle time with a series of procedural changes as shown in Helsinki.[2] They did this by making 12 simple changes to the way these patients were managed in both the pre hospital and acute phase of their care. This is useful as a template but it does not translate directly to the NHS which is organisationally different. Organisational barriers can be reduced in order to achieve a rapid patient flow through the acute service but this requires more negotiation than it might abroad since the demands upon acute services in this country are so high. However, it is possible to reduce the door to needle times so they are consistently below 40 minutes, as evidenced by other acute stroke units within our region.

\section{Baseline measurement}

The stroke database was searched (this is where door to needle times are captured) for each patient who was thrombolysed. A subanalysis was performed by reviewing the figures throughout their journey for the time it took the patient to get to A\&E from initial symptom onset, then from door to CT and then door to needle. 
BMJ Quality Improvement Reports

Times from both in and out of hours were reviewed.

The average door to needle time was 74.7 minutes (figure 1), and was longer over the weekends and out of hours. This was expected since this is when telemedicine was used by the on call staff which takes longer to use. Hours when the timings across the whole journey were reviewed were found to be much quicker because the management would be led by the on site stroke physician (figure 3). It was clear from the baseline measurements that there was a long period of time from the CT scan being performed to the drug being administered. This time also takes into account the review of the brain scan (which sometimes requires a radiologists' opinion), taking the patient to casualty, making the decision to give the drug after ruling out contraindications, and then consenting the patient/gaining assent from the family. There are possible barriers within each step of the process so the junior doctors "mapped the pathway" to help me identify where these were. This involved them writing down the exact process that they went through went seeing each stroke patient, how long each step took, and recording any barriers or delays to the journey.

See supplementary file: ds3957.xlsx - "Door to Needle Timings 2012 - $2014 "$

\section{Design}

After reviewing the figures and looking at the existing pathway, it was clear that a complex intervention would be necessary. Through reading the existing literature and combining this with previous experience, the following intervention was formulated: to introduce an ambulance pre-alert, re-write the existing pathways so they were more clear to follow, and provide education and training to the doctors and nurses within the hospital. There had already been a change in service where the thrombolytic agent could be given to the patient while in the CT scanner, so implementation of this was encouraged where possible.

Introducing an ambulance pre-alert meant that the ambulance crew would (within hours) call the stroke phone and warn us that a patient that potentially required thrombolysis was being brought in. They were asked to tell us the following information: name, date of birth, any seizure at onset, medications, blood pressure, and blood sugar. It was initially hoped that casualty department staff could use a checklist to get this information for all patients when they rang the call in to A\&E, but they were not willing to take on what they perceived to be an additional burden of work. The ambulance service agreed the second approach (introducing a pre-alert) and implemented this for some (though not all) patients over time.

Rewriting the existing pathways and clarifying the process was a more straightforward task, and these were presented at junior doctor induction meetings and included in the junior doctor handbook. Education was delivered at grand round, departmental, and A\&E meetings in the form of Powerpoint presentations and reminders. The information technology department added a message to the desk top of all the trust computers. The drug cupboard in the CT scanner was kept stocked for rapid administration of alteplase.

\section{Strategy}

This was carried out as an ongoing series of changes with regular review within team meetings.

PDSA cycle 1: Hospitals within our regional network which were using the pre-alerts were contacted and asked about how they had implemented the change and what problems they had encountered. This was discussed at a regional stakeholders meeting and an agreement was made with the local ambulance service. A checklist was formulated and circulated for feedback and the ambulance service informed their teams. The issue that arose from this was that $A \& E$ nursing staff were not happy to gather this extra information as they felt that the priority was ensuring training for all the staff involved in the service. We agreed that the ambulance service would ring the stroke phone directly and that this would only happen within hours.

PDSA cycle 2: Teaching and training sessions were held for all the staff involved in delivering the service. The main issue was one of attendance of those who were key to managing the services.

Teaching was given at the medical grand round, departmental, and A\&E meetings. In these sessions the value in shortening the door to needle times was reiterated and feedback was gathered on the audience's experience of using the service.

PDSA cycle 3: A "Time is Brain" campaign was advertised on the intranet while continuing to raise awareness in a wider forum within the hospital. This meant that the information technology department put a message on the desktop of all the trust computers informing staff that the project was ongoing and why it was necessary.

PDSA cycle 4: The pathway paperwork was revised and the formatting changed to make it easier to follow. This was put on the trust intranet after circulating it to the junior doctors. This was then presented at the junior doctor induction meetings and added to the junior doctor handbook.

The case notes of outliers was reviewed to see if there were any reasons for these to have been delayed in comparison to the other patients. Re-audit was carried out at regular intervals to present to the team and the wider hospital.

\section{Results}

The figures were re-audited (see attached) which showed a clear improvement over time. The median door to needle dropped from 65.5 minutes in 2012 to 49 minutes in 2014 . This was comprised of a general reduction in the time taken to traverse the pathway. The times continued to be much shorter within hours when the stroke team was on site.

Other metrics such as staff engagement with the stroke service and level of understanding around managing acute strokes is more difficult to measure. However, we did receive good feedback while going through the process, particularly in responses to the teaching delivered. 


\section{BMJ Quality Improvement Reports}

See supplementary file: ds3958.xlsx - "Door to Needle Times 2012-14"

\section{Lessons and limitations}

Apart from practical challenges, promoting a cultural shift in mindset was an extremely difficult thing to do. Part of this was simply due to the overwhelming number of conflicting commitments that health care professionals are subject to. This is particularly true for accident and emergency staff who are regularly given new targets and protocols from all the specialties to follow in acutely unwell patients. Convincing them that change was necessary was possible but that this had to stay within the constraints of not asking anyone to add to their workload. Requests to use staff and pathways more creatively were met with considerable resistance. Another major constraint was the constantly changing workforce of junior and locum doctors; any teaching/training had to be frequently repeated but other than this it was a case of hoping that they would consult the intranet for protocols or the stroke trained members of staff when they encountered unfamilar situations. A service which incorporated several different systems (thrombolysis via telemedicine, on site stroke physicians, casualty doctors) was both harder to analyse initially and to change.

In terms of change management, I began to understand the nature of change as a step by step process and just how long this can take. It also gave me a greater depth of understanding into how systems work within a hospital organisation and working across specialties. Lessons learnt were predominantly around leadership and behavioural change. It became clear quickly that behaviour was more likely to change if there was a visible example of good practice. There was also a feel of loss of momentum if the project was not given a regular slot for discussion in departmental or hospital meetings.

\section{Conclusion}

A complex intervention carried out in a step by step manner is effective for reduction of door to needle time for stroke thrombolysis. The elements incorporated within this were pathway mapping and analysis, the identification of barriers, and problem solving focussed on these and education around the management of stroke. The part of this project that really enabled change was the regular review and discussion of the experiences of those working within the service which allowed for evolution.

\section{References}

1. Tissue Plasminogen Activator for Acute Ischaemic Stroke. The NINDS Stroke Study Group. NEJM 1995; 333:1581-8.

2. Meretoja A, Weir L, Ugalde M, Yassi N, Yan B, Hand P et al. Helsinki Model Cut Stroke Thrombolysis Delays to 25 Minutes in Melbourne in only Four Months. Neurology 2013;81(12):1071-6.
There are no conflicts of interest.

\section{Acknowledgements}

Ruth Connolly, general manager A\&E. 